\title{
Frequency and temperature control for complex system engineering in optoelectronics and electronics: an overview
}

\author{
Patrice Salzenstein ${ }^{*}$
}

Centre National de la Recherche Scientifique (CNRS), Franche Comté Electronique Mécanique Thermique Optique Sciences et Technologies (FEMTO-ST) Institute, Université de Bourgogne-Franche-Comté (UBFC), 15B avenue des Montboucons, 25030 Besançon, France.

Received: 8 January 2020 / Accepted: 3 May 2020

\begin{abstract}
To take advantage of the physical principles of determining parameters, such as frequency stability, noise and also alignment of optical signals, it is necessary to control complex systems. This work allows explaining it through various concrete cases such as the determination of phase noise of microwave oscillators, the control of the temperature of the manufacturing process of optical components. We also discuss the estimation of the uncertainty associated with the measurement results, as it is fundamental to control the error range.
\end{abstract}

Keywords: Frequency stability / Phase noise / Temperature / Optoelectronics / Electronics / Resonator / Oscillator

\section{Introduction}

In this paper we would like to return to the main theme that guided the various researches. It may seem quite large, but everything has been logically linked since this work on phase noise measurements on quartz oscillators, until the development of optic resonators, and their integration in optoelectronic oscillators. It is then necessary to characterize these optoelectronic oscillators. The link between all these research works is the concern of the control of the frequencies and the temperature in particular. The common structure of this research is certainly the need to control relatively complex systems. These are not only oscillators, but also systems that measure their performance. We are thinking in particular of phase noise measurement benches, or frequency stability measurement benches.

After this introduction, the rest of this paper is organized as follows. We have basically split our article into three grade parts. First of all, part 2 of this paper is devoted more specifically to the quartz oscillators and benches, which have been necessary to develop or simply to use to characterize their performances. Part 3 is devoted to optoelectronic oscillators. This begins with the optical resonators and then the optoelectronic oscillators themselves, and finally we discuss the methods of stabilization of

\footnotetext{
* e-mail: patrice.salzenstein@femto-st.fr
}

lasers and then the characterization of the performances of this type of oscillators. Part 4 is dedicated to the need of evaluating uncertainties and optimization.

\section{Dependence of quartz oscillators performance to frequency and temperature}

We mainly focused on the work on quartz oscillators in the last decade. We did not go back on the epic of technological developments on quartz. Piezoelectricity and quartz oscillators have their origins in the works of Jules Lissajous [1], as well as those of Jacques and Pierre Curie [2,3]. To consult the history until the recent period, it is possible to refer to a previous article [4]. We are now doing a good step forward to arrive in the recent period.

Perhaps it is useful to recall what a quartz oscillator is to better understand the issues. We have a quartz resonator, which can be of different types. There is also an amplifying element so that the gain in the oscillation loop is greater than 1 . It is necessary to compensate for the losses in the resonator, by sufficiently amplifying the electrical signal. There is also a phase shifter to ensure phase matching and a directional coupler or splitter to be able to take a part of the signal from the oscillation loop.

In Figure 1, we have very schematically represented a quartz oscillator. We recollect what we mentioned earlier, namely, the quartz resonator with its power supply, the amplifier electro, the phase shifter, the directional coupler, 


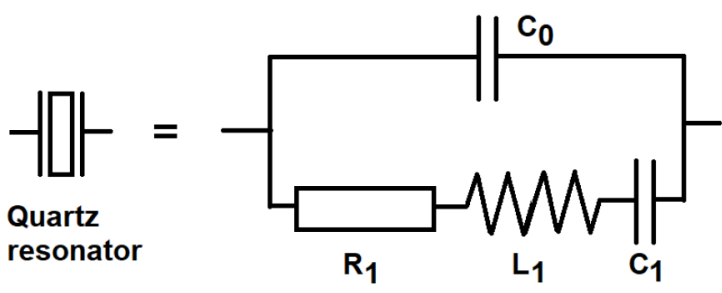

(a)

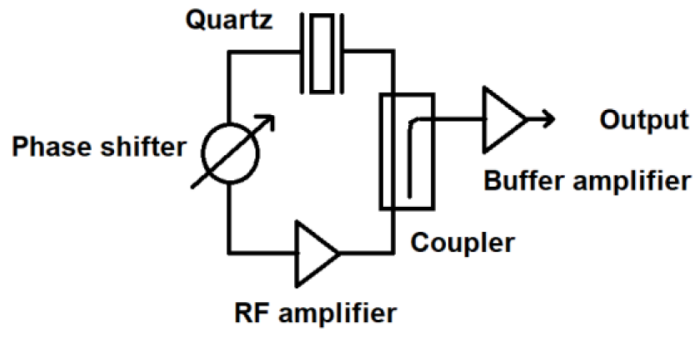

(b)

Figure 1. Principle of a quartz oscillator. A quartz crystal is equivalent to an electrical network with a low impedance in series and a parallel high impedance resonance. R1: resistance, L1: self, C0 and C1: capacitances. (a) Symbol and equivalent circuit of a quartz. (b) Quartz oscillator.

the buffer amplifier, and the output filter. It must also be ensured that the signal remains low noise when it is distributed [5]. To reject harmonics, signal is filtered before the input and after multiplication and amplification. To ensure the good performances of the best quartz oscillators, it is necessary to have a double oven structure to prevent from thermal variations inside the oscillator. It means there is an internal oven control electronic board and an external board for the second oven.

Regarding the phase noise of the oscillator, it will be mainly related to the phase noise of the resonator, and also to the amplifier present in the loop. Different types of noise give different slopes depending on their type and the contribution of the effects considered. For resonator phase noise measurement, please refer to references [6]. The best performances in terms of noise were measured on BVA type crystal oscillators. Ten years ago, it was proved that flicker noise floors as low as $2.5 \times 10^{-14}$ could be reached within seconds to estimate Allan variance as a function of integration time. These results were published in 2010 [7-9], and reconfirmed thereafter [4].

Figure 2 shows the best performances obtained for Allan variance on a quartz at $5 \mathrm{MHz}$. We have seen that quartz oscillators could have some interesting ultimate performance in the state of the art. However, there has been no noticeable progress in the last decade. Moreover, as research efforts are probably less now, compared to what has been the case a few decades ago, it can be assumed that there will be no marked improvement in performance. These are anyway caused by the quartz resonator itself. This can be caused by Drive Level Dependence in Quartz Crystal Resonators [10].

It was timely to ask the question of the performance that can be achieved using optoelectronic oscillators. This will be investigated in the next part of this article.

\section{Sensitivity of optoelectronic oscillators to temperature and environment}

Optoelectronic oscillators (OEO) were proposed by a Californian team 25 yr ago [11]. The idea of pushing the performance of this type of oscillator is to know if they can one day be good candidates to replace the oscillators based on quartz. If we can have optoelectronic oscillators, and

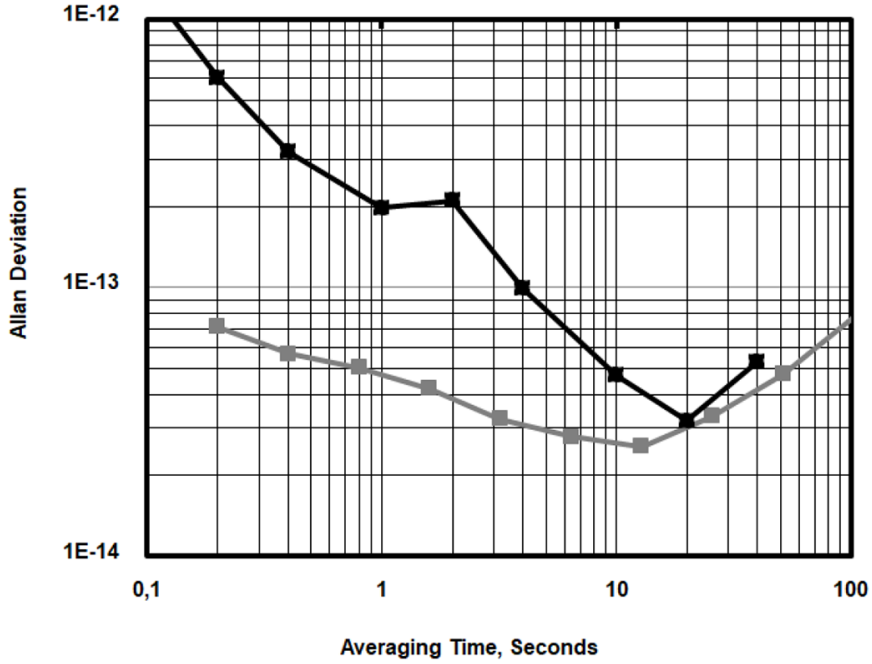

Figure 2. Best performances obtained on a Quartz oscillator at $5 \mathrm{MHz}$. The flicker frequency modulation (FFM) floor is in the range of $2.5 \times 10^{-14}-3.2 \times 10^{-14}$ for 10 to $20 \mathrm{~s}$ integration time. In grey the best result measured by Dual Mixer Time Difference Multiplication [8], in black the confirmation of this performance measured with time interval analyzer (TIA) [10]. For the black curve, results are limited by the TIA for lower averaging time and by aging for upper averaging times.

they have very good performance in terms of phase noise, then it will be possible to consider using them, for example, for space applications. These oscillators may be less sensitive to certain parameters such as the acceleration that is necessary because spacecraft must be launched from Earth. It would also be less resistant to electromagnetic radiation. In space, satellites, rockets or a space station are necessarily subject to the consequences of solar flares. We can easily imagine that an optoelectronic system can be better protected than an electrical system. An embedded system that is based on optoelectronic components could therefore be more reliable. However, it is fundamental that the performance of such oscillators can compete with those of quartz oscillators. This is why an important part of the work was to seek to improve the performances in terms of phase noise, in particular for the Fourier frequency close to the carrier. 


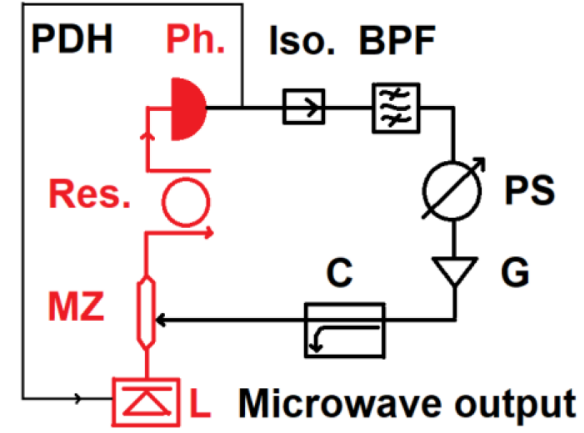

(a)

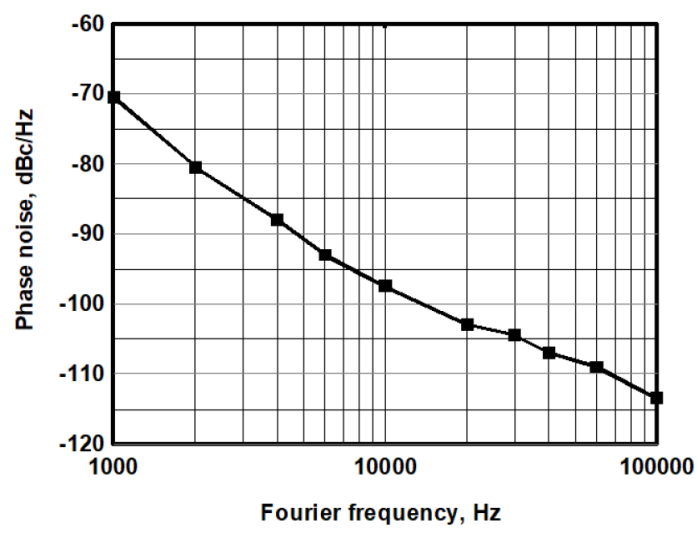

(b)

Figure 3. Optoelectronic oscillator schematized and its performances in terms of phase noise. (a) Optical elements and electrical elements are respectively drawn in red and black colours. L: laser, MZ: Mach Zehnder modulator, Res: optical resonator, Ph: photodetector, PDH: Pound Drever Hall, Iso: isolator, BPF: band pass filter, PS: phase shifter, G: microwave low noise amplifier, C: coupler. (b) Phase noise performance of an OEO based on an optical resonator.

The numerous applications involved in working with optoelectronic oscillators and needing a lower noise level for Fourier frequencies farther from the carrier, for example, for aeronautical applications or telecommunications, will not be considered here. It would be tedious in an article like this to consider all the possibilities offered by optoelectronic oscillators. We only say few words to remember that with the same configuration as these optoelectronic oscillators, by injecting more optical power, we can see that the nonlinear effects appear and make it possible to make frequency combs. What then becomes interesting is not the stability of the signal itself, but the stability of the difference between two consecutive optical signals, which can be improved, for other applications, including metrology.

The optical resonator can basically be of two types. It may be an optical delay line or an optical resonator:

- If it is an optical delay line, several possible modes will be in competition separated by a free spectral interval. To select a resonance mode, it is necessary to provide various additional elements. It is of course necessary to amplify the electrical signal after the photodetector. The amplifier is not ideal, it can naturally favour this or that mode of resonance. Moreover, we can add a band pass filter. If this band pass is selectively selective and covers a restricted range of frequencies, it is all the easier to favour the selection of a particular mode, if this filter is not ideal. The flatness being irregular, it will allow a greater natural selectivity of the resonance mode.

- If the resonator is an optical resonator. There are then several types of resonators possible. It may be a sphere $[12,13]$, but also a disk or what matters is the propagation of the signal at its periphery, either because the disk is of toroidal type [14], or because the radius of curvature of its slice is sufficiently small. To couple the optical signal to the optical resonator, it is necessary to ensure a coupling of the evanescent type [15-17]. It is then necessary to remain at an optimal distance from the resonator to allow both the transmission of a part of the signal but also the coupling to the resonator. With the aid of a photodetector, the difference between the signal transmitted and delayed in the resonator is detected [18]. The delay is even greater as the quality factor of the resonator is high [19]. It is also possible to directly transmit the signal via a double coupling of the optical resonator. The signal transmitted is then detected through the resonator.

It is also possible to use both an optical resonator and a delay line, or several delay lines to select modes. It is then a question of playing with the topology of the optoelectronic oscillator.

Figure 3 shows the main principle of an OEO and a typical curve showing the performances of such an oscillator based on an optical resonator. Pound Drever Hall (PDH) is a well-known technique for stabilizing the frequency of light emitted by a laser by means of locking it on the optical resonator $[20,21]$. As detailed in a previous work [22], it is possible to decrease the level of noise by using an additional optical delay line [23]. Then the noise at 1 and $10 \mathrm{kHz}$ from the carrier is respectively $42 \mathrm{~dB}$ and $28 \mathrm{~dB}$ lower.

The level of performance could best be obtained for optoelectronic oscillators. In this case, it is found that it is difficult to obtain better than $25-30 \mathrm{~dB}$ more than for the best quartz oscillators on the Fourier frequency segment between $10 \mathrm{~Hz}$ and $100 \mathrm{~Hz}$, for example. It is still too early to really consider that this type of optoelectronic oscillator could perform as well as the quartz oscillators.

Engineering work has been done to control, for example, the temperature rise of an annealing furnace for application to optical resonators [24-26]. More specifically, the electronic part is described in reference [27].

An example of the characterization of an optoelectronic oscillator is given in reference [28]. 
Table 1. Phase noise of an OEO with an output power of $+5 \mathrm{dBm}$ at $10.52 \mathrm{GHz}$.

\begin{tabular}{lll}
\hline $\begin{array}{l}\text { Offset to the } 10.52 \mathrm{GHz} \text { carrier } \\
\text { Fourier frequency }(\mathrm{Hz})\end{array}$ & $\begin{array}{l}\text { Measure with R\&S bench } \\
\text { Phase noise }(\mathrm{dBc} / \mathrm{Hz})\end{array}$ & $\begin{array}{l}\text { Measure with FEMTO-ST bench } \\
\text { Phase noise }(\mathrm{dBc} / \mathrm{Hz})\end{array}$ \\
\hline $2 \times 10^{3}$ & -100 & -100 \\
$4 \times 10^{3}$ & -109 & -112 \\
$6 \times 10^{3}$ & -115 & -118 \\
$10^{4}$ & -119 & -130 \\
$2 \times 10^{4}$ & -125 & -140 \\
$3 \times 10^{4}$ & -125 & -145 \\
$4 \times 10^{4}$ & -123 & -141 \\
\hline
\end{tabular}

\section{Uncertainty evaluation}

In this part, we will mainly focus on instrumental methods that have been used to estimate or measure the phase noise of quartz resonators, quartz oscillators and optoelectronic oscillators. It is not a question of precisely describing each of the methods, because this has already been published, as we will see. It is a question of looking more specifically at the uncertainty of measurement. Giving the noise level of the different types of oscillators without having a precise idea of the uncertainty associated with the measurement results, does not make sense.

For the quartz resonator noise measurement benches, work has given both a description of this type of instrument and the uncertainty that could be expected [29].

To measure the phase noise of an oscillator, whether electric or optoelectronic, there are commercial banks. Instrument manufacturers systematically give uncertainty. Of course, this uncertainty must still be degraded by the user because the instruments are not used in the same way in each laboratory or factory, compared to what the manufacturer recommends and was able to measure by itself.

Instruments make it possible to directly measure the noise of a signal generated by an oscillator while for others, there are methods for the type of triangulation. We compare several pairs of oscillators between them and we deduce the respective weight of each oscillator in its contribution to the noise of the oscillator pair, in each case.

By way of illustration, we remind about the estimation of the uncertainties associated with measurements in the case of different tested devices. Uncertainties are estimated according to modern methods [30,31]. For the measurement of oscillators, it is advisable to refer to a series of publications on an optoelectronic measuring bench [32-34]. For electrical measurements, in the microwave domain, we can also consult recent work that estimates the level of uncertainty [35].

As an illustration, we choose an OEO delivering an output signal of $+5 \mathrm{dBm}$ at $10.52 \mathrm{GHz}$. It is constituted of a RIO Laser (driven by a $125 \mathrm{~mA}$ signal) before a modulator, a $4 \mathrm{~km}$ optical fibre delay line, a DSC40S Discovery photodetector, a $+54 \mathrm{~dB}$ gain amplifiers for microwave signal, an
X-band filter, an ARRA passive phase shifter and a buffer amplifier (AML812-1901) at the lateral arm of a microwave coupler to extract the output microwave signal. Results are given in Table 1.

Measurement results differ between the same OEO measured by the commercial Rohde \& Schwarz instrument and FEMTO-ST system: it is due to the noise limitation at $-125 \mathrm{dBc} / \mathrm{Hz}$ for the $\mathrm{R} \& \mathrm{~S}$ instrument. The OEO presents a minimum of $-145 \mathrm{dBc} / \mathrm{Hz}$ at $3 \times 10^{4} \mathrm{~Hz}$ from the $\mathrm{X}$-band carrier.

The uncertainty for this type of phase noise measurement bench is typically of the order of $2 \mathrm{~dB}$ at two sigmas.

This explains the variations between the results on the same oscillator with a commercial bench and a bench created in the laboratory.

\section{Conclusion}

In this paper, we have attempted to show the need for temperature and frequency control for some types of oscillators which are relatively complex systems. We have seen in particular that we have been able to obtain state-ofthe-art performances on quartz oscillators delivering a signal at $5 \mathrm{MHz}$. On the other hand, we explored the case of optoelectronic oscillators to estimate to what extent it would be possible to use them to replace quartz. But the performances obtained to date on the OEO remain too weak and it is not possible to use them in oscillators alone. However, other applications are promising such as those which use frequency combs by increasing the power emitted by the laser and by taking advantage of nonlinear effects.

This work was sponsored by Agence Nationale de la Recherche grant \#ANR2010BLAN031202, Ministère des Affaires étrangères et $\mathrm{du}$ Développement International (MAEDI) and Campus France grant \#885706G 2016.

\section{References}

1. J. Lissajous, Mémoire sur l'étude optique des mouvements vibratoires, Comptes Rendus 44, 727 (1857) 
2. J. Curie, P. Curie, Développement par pression de l'électricité polaire dans les cristaux hémièdres à faces inclinées. Comptes Rendus 91, 294 (1880)

3. J. Curie, P. Curie, Déformations électrique du quartz. Comptes Rendus 95, 914-197 (1882)

4. P. Salzenstein, Recent progress in the performances of ultrastable Quartz resonators and oscillators, Int. J. Sim. Mult. Des. Optim. 7, A8 (2016)

5. P. Salzenstein, N. Cholley, A. Kuna, P. Abbé, F. LardetVieudrin, L. Sojdr, J. Chauvin, Distributed amplified ultrastable signal quartz oscillator based, Measurement 45, 1937-1939 (2012)

6. P. Salzenstein, A. Kuna, L. Sojdr, F. Sthal, N. Cholley, F. Lefebvre, Frequency stability measurements of ultra-stable BVA resonators and oscillators, Electron. Lett. 46, 686-688 (2010)

7. A. Kuna, J. Cermak, L. Sojdr, P. Salzenstein, F. Lefebvre, Lowest flicker-frequency floor measured on BVA oscillators, IEEE Trans. UFFC 57, 548-551 (2010)

8. P. Salzenstein, A. Kuna, L. Sojdr, J. Chauvin, Significant step in ultra high stability quartz crystal oscillators, Electron. Lett. 46, 1433-1434 (2010)

9. H. Dyball, Dropping through the floor, Electron. Lett. 46, 1411 (2010)

10. R. Brendel, M. Addouche, P. Salzenstein, E. Rubiola, Y. Shmaliy, Drive level dependence in quartz crystal resonators at low drive levels: a review, IEE Conf. Pub. 2004, 11-18 (2004)

11. X.S. Yao, L. Maleki, Optoelectronic microwave oscillator, J. Opt. Soc. Am. B 13, 1725-1735 (1996)

12. M. Mortier, P. Goldner, P. Feron, G.M. Stephan, H. Xu, Z.P. Cai, New fluoride glasses for laser applications, J. Non-Cryst. Sol. 326-327, 505-509 (2003)

13. A. Chiasera, Y. Dumeige, P. Féron, M. Ferrari, Y. Jestin, G. Nunzi Conti, S. Pelli, S. Soria, G.C. Righini, Spherical whispering-gallery-mode microresonators, Laser Photon. Rev. 51, 457-482 (2010)

14. D.K. Armani, T.J. Kippenberg, S.M. Spillane, K.J. Vahala, Ultra-high-Q toroid microcavity on a chip, Nature 421, 925-928 (2003)

15. Y. Dumeige, S. Trebaol, L. Ghisa, T.K.N. Nguyen, H. Tavernier, P. Feron, Determination of coupling regime of high-Q resonators and optical gain of highly selective amplifiers, J. Opt. Soc. Am. B 25, 2073-2080 (2008)

16. P. Salzenstein, M. Mortier, H. Sérier-Brault, R. Henriet, A. Coillet, Y.K. Chembo, A. Rasoloniaina, Y. Dumeige, P. Féron, Coupling of high quality factor optical resonators, Phys. Scr. T157, 014024 (2013)

17. D. Bassir, P. Salzenstein, Optimization of fiber to resonator coupling, Proc. SPIE 11357, 113571P (2020)

18. K. Volyanskiy, P. Salzenstein, H. Tavernier, M. Pogurmirskiy, Y.K. Chembo, L. Larger, Compact Optoelectronic Microwave Oscillators using Ultra-High Q Whispering Gallery Mode Disk-Resonators and Phase Modulation, Opt. Express 18, 22358-22363 (2010)

19. R. Henriet, P. Salzenstein, D. Ristic, A. Coillet, M. Mortier, A. Rasoloniaina, K. Saleh, G. Cibiel, Y. Dumeige, M. Ferrari, Y.K. Chembo, O. Llopis, P. Féron, High quality factor optical resonators, Phys. Scr. T162, 014032 (2014)
20. R.W.P. Drever, J.L. Hall, F.V. Kowalski, J. Hough, G.M. Ford, A.J. Munley, H. Ward, Laser phase and frequency stabilization using an optical resonator, Appl. Phys. B 31, 97-105 (1983)

21. E. Black, An introduction to Pound-Drever-Hall laser frequency stabilization, Am. J. Phys. 69, 79-87 (2001)

22. K. Saleh, R. Henriet, S. Diallo, G. Lin, R. Martinenghi, I.V. Balakireva, P. Salzenstein, A. Coillet, Y.K. Chembo, Phase noise performance comparison between optoelectronic oscillators based on optical delay lines and whispering gallery mode resonators, Opt. Express 22, 32158-32173 (2014)

23. R.T. Logan, L. Maleki, M. Shadaram, Stabilization of oscillator phase using a fiber-optic delayline, Proceedings of the 45th Annual Symposium on Frequency Control (IEEE, 1991), pp. 508-512 (1991)

24. M. Zarubin, P. Salzenstein, Temperature controlled optical resonator process for optoelectronic oscillator application, Proc. SPIE 9503, 950311 (2015)

25. P. Salzenstein, S. Diallo, M. Zarubin, Electrically driven thermal annealing set-up dedicated to high quality factor optical resonator fabrication, J. Power Technol. 98, 198-201 (2018)

26. P. Salzenstein, D. Bassir, M. Zarubin, Optimized oven for optical resonator heating process, Proc. SPIE 11334, 1133406 (2019)

27. P. Salzenstein, M. Zarubin, Electronics improvements for optical resonators fabrication, Proc. SPIE 10814, 1081418 (2018)

28. P. Salzenstein, An example of design, optimization, stabilization and noise performances of resonator based optoelectronic oscillators, Int. J. Sim. Mult. Des. Optim. 10, A2 (2019)

29. F. Sthal, J. Imbaud, X. Vacheret, P. Salzenstein, G. Cibiel, S. Galliou, Computation method for the short-term stability of quartz crystal resonators obtained from passive phase noise measures, IEEE Trans. UFFC 60, 1530-1532 (2013)

30. R. Kacker, K.D. Sommer, R. Kessel, Evolution of modern approaches to express uncertainty in measurement, Metrologia 44, 513-529 (2007)

31. W.-K. Lee, D.-H. Yu, C.Y. Park, J. Mun, The uncertainty associated with the weighted mean frequency of a phasestabilized signal with white phase noise, Metrologia 47, 24-32 (2010)

32. P. Salzenstein, E. Pavlyuchenko, A. Hmima, N. Cholley, M. Zarubin, S. Galliou, Y.K. Chembo, L. Larger, Estimation of the uncertainty for a phase noise optoelectronic metrology system, Phys. Scr. T 149, 014025 (2012)

33. P. Salzenstein, E. Pavlyuchenko, Modern approach for estimating uncertainty of a precision optoelectronic phase noise measurement, 2013 International Conference on Advanced Optoelectronics and Lasers (CAOL), Sudak, Ukraine, pp. 340-341 (2013)

34. E. Pavlyuchenko, P. Salzenstein, Application of modern method of calculating uncertainty to microwaves and optoelectronics, 2014 International Conference on Laser Optics, Saint Petersburg, Russia, June 30 2014-July 4

35. P. Salzenstein, T.Y. Wu, Uncertainty analysis for a phasedetector based phase noise measurement system, Measurement 85, 118-123 (2016) 